through the trochar tube of the encephaloscope for 54 hours. By comparing preradiation and post-radiation carotid dangiograms of the tumor, we could observe that the tumor became smaller and that the radium insertion had its usefullness in some respects. Radium is not the best source, however, and we wish to have a chance to use some other good source in our method of treatment of inoperable deep seated brain tumors by means of the stereotaxic insertion.

\title{
S-2. Infusion Chemotherapy for Brain Tumors in Our Clinic
}

\author{
Hajime HaNDa and Kazuo MoRI \\ Dept. of Neurosurgery, Kyoto University School of Medicine
}

Antimetabolite solution was injected continuously through a fine $(0.7 \mathrm{~mm}$ in diameter) Teflon catheter into unobstructed artery to produce high regional drug concentration for several days to weeks. Methotrexate $(50 \mathrm{mg} / \mathrm{day})$ with antidote therapy by systemic administration of citrovorum factor was used preferentially and total doses of MTX were at the range of 500-1500 mg.

Eleven patients treated in this manner were far advanced cases ( 10 cases of glioblastoma of cerebral hemisphere and one case of midcerebellar tumor). Most of them had recurrences of tumor and did not responded to the radiotherapy and catheter was introduced either into internal carotid or vertebral artery depending upon the location of pathology.

Since all of the subjects treated in our clinic were near the terminal stage at the time of infusion, it might be too early to evaluate fully the benefits of this treatment. However, seven cases out of these eleven, died within three weeks after the therapy with complications mostly attributable either to depressed function of bone marrow or brain edema.

The results of radiation treatment of 285 cases of brain tumor were also presented. 